\title{
ACCIDENT ANALYSIS OF LOW PRESSURE GAS DISTRIBUTION SYSTEMS
}

\author{
Alexander Shkarovskiy ${ }^{1,2}$, Maciej Kotuła ${ }^{3}$ \\ ${ }^{1}$ Saint Petersburg State University of Architecture and Civil Engineering \\ Vtoraja Krasnoarmejskaja ul., 4, St. Petersburg, 190005, Russia \\ ${ }^{2}$ Koszalin University of Technology \\ 2 Sniadeckich St., 75-453 Koszalin, Poland \\ ${ }^{3}$ Gas Supply Company in Kołobrzeg, Polish Gas Company Ltd. \\ 30 Koszalińska St., 78-100 Kołobrzeg, Poland \\ ${ }^{1}$ szkarowski@mail.ru
}

\begin{abstract}
In terms of safety, gas supply represents a highly important area of science and engineering. On the one hand, gas is a very convenient primary energy source for consumers. On the other hand, gas can be extremely dangerous for life and health of people, if strict requirements to design, construction and operation of gas supply systems are not complied with. The article reviews an accident that occurred in a small Polish city in 2010 . We analyzed data from the Prosecutor's Office and relevant publications and questioned workers of the gas supply company. The article describes how an unlucky train of events resulted in a tragedy. The main circumstances were an unacceptable change in the gas distribution plant equipment and violation of operating rules, which resulted in an unauthorized pressure increase in a district network and internal systems designed for low pressure. We analyzed follow-up technical and administrative measures taken regarding the Polish gas supply system and compared some peculiarities of Polish and Russian gas technologies. The main purpose of the analysis is to prevent such accidents in future.
\end{abstract}

\section{Keywords}

Gas supply, safety, gas distribution plant, equipment, operation, pressure increase.

\section{Introduction}

The concept and main components of gas supply systems are similar in most countries. Therefore, accidents are also similar in their reasons, progress and consequences. The article presents results of an analysis of one such accident. Experts in design, construction and operation of gas supply systems can assess, on their own, how a combination of circumstances (however improbable) can result in a tragedy. This will probably allow avoiding such events through simple design, technological and administrative measures (Ansari et al., 2017).

\section{Subject, objectives and methods}

The accident analyzed in the article occurred on November 30, 2010 in small Polish city Zielona Góra with population about 140 thousand people, situated in the Lubusz province in Western Poland, close to the border with Germany. We can judge about the beginning of the accident based only on subjective and highly emotional statements of those affected who claimed that "...gas stoves in apartments started exploding one by one" (Super Express, 2010). If we assess those statements objectively, it was enough to try to light a gas-range burner 
for a breakout of heavy fire that would be impossible to put out. Such fire was particularly the case when oven burners were attempted to be lit, where a burst of flame actually differed little from an explosion (Fu et al., 2018).

Let us think about the reasons why the fire could not be put out and what followed. Generally, we turn a stove knob, already holding a lit match or a stove lighter in the other hand, or (in modern models) simultaneously activating a spark igniter. In case of a strong burst of flame, a person jumps back, intuitively protecting himself from the flame. Obviously, the gas knob is left open. In modern ovens, a flame sensor can activate, but the outcome is the same. Panic prevents people from reaching the stove to close the gas knob or shut off the gas tap, the latter quite often being even less easy to reach (Kim et al., 2018).

The events swept three neighborhoods - Pomorskie (Pomeranian), Śląskie (Silesian) and Raculka, where the explosions resulted in three fires. Two of them were extinguished rather quickly, but the third one, which swept an apartment on the 8th floor in the Pomorskie (Pomeranian) neighborhood, followed a tragic scenario. Firemen fought against it for several hours and saved a woman cut off by the fire. However, a fifty-year-old man died in the fire. He probably had lost consciousness at the time of the explosion. 6,500 people were evacuated from the houses affected by the accident. One can easily imagine their mental state amidst the fires and the howl of fire-engines (Tymchik et al., 2018).

According to the Minister of the Interior and Administration, no such large-scale accident had ever happened in Poland. In terms of the number of victims, there had been even more tragic isolated accidents affecting an apartment, a stairwell or a residential building. Still, if we estimate the area affected by the accident, the number of houses and evacuated people, one cannot but agree with such estimation. The containment of the accident involved: 500 policemen (53 vehicles), 177 professional and volunteer firemen (47 vehicles), 35 city guards (two vehicles), 17 ambulance health workers, 100 emergency gas service specialists (50 vehicles), three emergency power supply service workers (1 vehicle), 10 specialists from the crisis management center, along with 17 city buses (Vasista, Alsudairi, 2018).

\section{Results and discussion}

\section{Preliminary analysis}

The reason of the accident remained unknown for a long time, as the investigation went on. However, it was not very difficult for specialists to make a preliminary conclusion that medium-pressure gas started to flow to the low-pressure gas distribution system. It started from a gas distribution plant (GDP) and went to the street network, and then to internal building systems.

When the gas supply system under consideration (1970-1980s) was constructed, gasification of populated areas developed in Poland similarly to that in the majority of other countries. Medium-pressure gas was supplied to stand-alone GDPs, and the built-up area was covered by a network of low-pressure gas pipelines. Such layout was considered to be safer. Considering that the maximum pressure upstream of household gas appliances should not exceed $2.5 \mathrm{kPa}$, the entire network was usually designed in the operating pressure range of $2.0 \ldots 2.5 \mathrm{kPa}$.

Pipelines and equipment of such systems were under pressure of several bars, which was hundreds of times higher. Many system elements, such as taps, seals, supply hoses, might just fail to operate, which would result in gas contamination of premises, gas accumulation in ovens, etc. Suffice it to say that gas stoves have a certificate of gas density at pressure up to $3.0 \mathrm{kPa}$, and gas meters up to $5.0 \mathrm{kPa}$. What happened when a person tried to light a burner was briefly analyzed above.

The very fact of medium-pressure gas entering the low-pressure gas system was clear to the specialists. Still, without necessary technical documentation, it was difficult even for scientists and skilled professionals in design and operation of gas supply systems to determine clear reasons.

The GDP design in any country would not allow medium pressure-gas to enter the exhaust gas pipeline. At least three devices prevent such entry: a pressure regulator itself and two safety valves - a safety shut-off valve and a safety discharge valve. Operating together, they should prevent outlet pressure from exceeding the established level (which in Russia, as a rule, is $20 \%$ higher than the maximum allowable level). The shut-off valve actuates when this happens. The discharge valve is adjusted for two times less value. This prevents an unjustified interruption of gas supply, which would be unavoidable at the shut-off device actuation.

There are few reasons for safety devices to actuate. Sometimes, at dramatic changes of the inlet pressure, dynamic performance of the pressure regulator may prove insufficient. Still, contamination of the pressure regulator valve seat is a more frequent reason - it should close completely if no gas is used, but this does not happen. The pressure regulator membrane can be damaged as well. The maximum pressure in low-pressure systems at the time of construction was $5 \mathrm{kPa}$. That is why, in any case, the shut-off valve should have actuated at a pressure not exceeding $6 \mathrm{kPa}$. Then what happened?

\section{Prosecutor's Office data}

Only at the end of the investigation when access to the documents was provided, specialists were able to begin a scientific analysis of accident causes. The authors of the article present their version of such analysis. In some cases, we are forced to deal with strict legal wordings and long sentences typical for Polish language, thus literal translation is complicated.

The first one of those wordings is the very title of the message dated January 18, 2013, posted on the official website of the Regional Prosecutor's Office: "The indictment against the gas distribution network master in the case of gas explosion in Zielona Góra neighborhood" (Prokuratura Okrégowa, 2013). Thus, the prosecutor's investigation translates the search for causes into the field of the human factor. Literal translation of the message is 
impossible in principle, nor required for the goals of the analysis. However, we should stick to the common logic of the message.

The prosecutor submitted the indictment against the gas distribution network master to court. The indictment states that, being responsible for modernization of the gas distribution plant, he tolerated an incorrect connection of GDP elements and used improper materials. His actions resulted in the GDP failure to operate, which manifested in the fact that exceedingly high-pressure gas entered the gas distribution system, leading to de-pressurization of the GDP and network that supplied gas to individual consumers. Consequently, the actions of the person accused led to the natural gas explosion in one of the buildings, thus largely threatening lives and health of many citizens, and their property.

The investigation found that on 30.11.2010 an accident involving the pressure regulator occurred. Unacceptably high gas pressure resulted in de-pressurization of internal systems in the apartments and uncontrolled gas leaks. As a result, there were heavy bursts of flame and so-called (in the original language) explosions of stoves at attempts to use those, along with the accumulation of large amounts of gas in the premises.

In order to determine causes of the accident at the GDP, developing under this scenario, the investigation turned to experts. They provided several causes in their expert opinion:

- reduced diameter of the discharge pipeline;

- improper pipeline laying (siphon pipeline laying);

- no protection against precipitation;

- failure of the standby line shut-off valve (the valve trigger did not affect the shut-off mechanism).

Those three pieces of the Prosecutor's Office message provide a great deal of essential information to be analyzed. It goes without saying that the core reason for the tragedy was the failure of the pressure regulator. However, such equipment failures do happen in the gas supply industry, like in any other engineering field. No tragic events happen at such failures, as gas distribution plants have a range of safety systems briefly described above (Wang, Yang, 2018).

Note the reference to the failure of the shut-off valve particularly in the standby line. This indicates that the main pressure reduction line was simply turned off. Brief information on the layout of the gas distribution plant designed more than 40 years ago is needed at this point. The GDP was constructed based on a two-line pressure reduction layout (Figure 1). Both pressure reduction lines are placed in the GDP building; they are fully functional and identical but for the equipment settings. They share only inlet and outlet shut-off and discharge units located outside the building, and the safety discharge valve.

\section{Human factor}

The authors had the opportunity to talk to the employees of this gas supply company. It turned out that at first the failure of the shut-off valve to actuate was named as the reason of the accident (although as rumor only). The materials of the prosecutor's investigation confirmed this assumption. However, based on the information received from the experts, the accident could be caused by an array of reasons that occurred simultaneously against the laws of probability.

The faults in GDP operation occurred during routine modernization of the equipment, that was performed through the efforts of the gas supply company. During the works, it was established that the safety shut-off valve on the main pressure reduction line did not operate properly. It was removed and placed under adjustment. The GDP was switched to the standby line for a long time.

It was this line where malfunction of the pressure regulator occurred. Most likely, it was due to a damaged membrane. This ultimately led to a spike in pressure downstream of the pressure regulator. An emergency situation occurred (which was still standard one), and

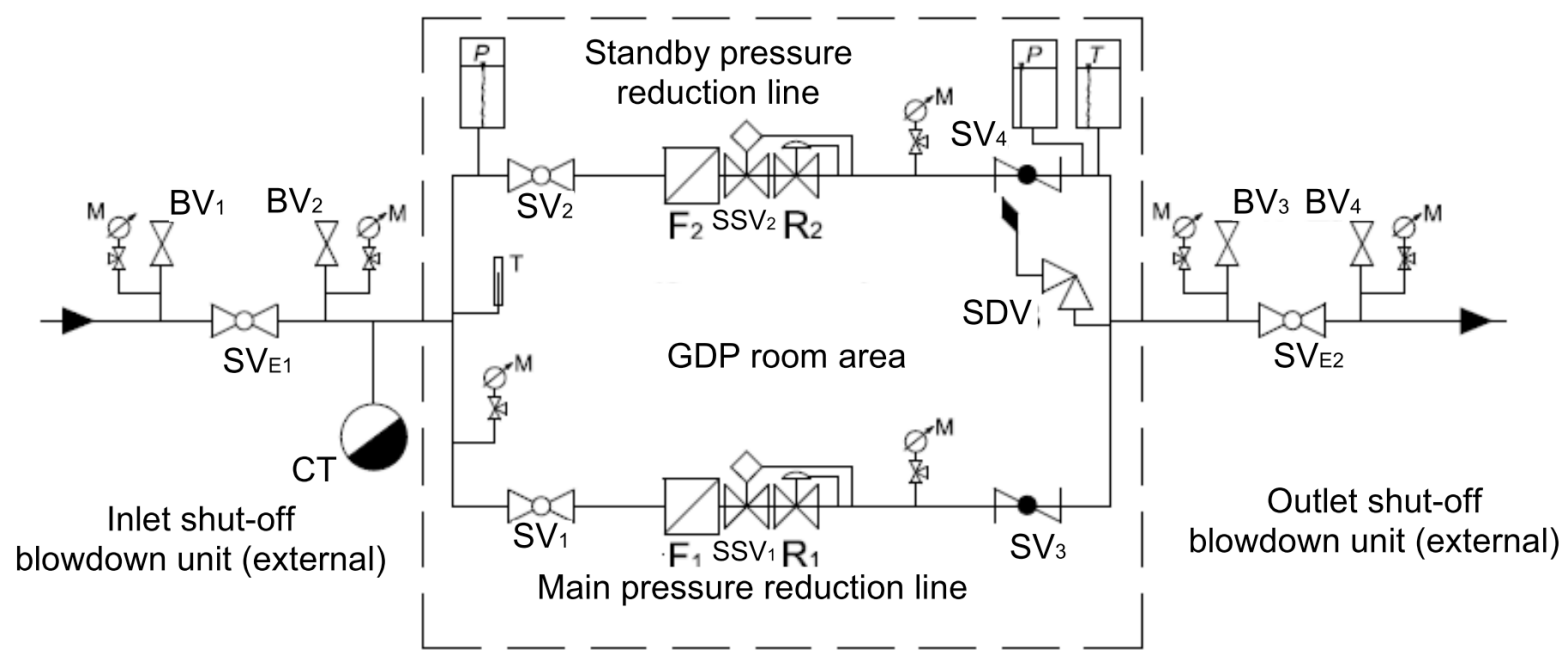

Figure 1. Layout of the low-pressure GDP with two pressure reduction lines:

SV - external shut-off valve; BV — blowdown valve; SV — shut-off valve; F — filter; SSV — safety shut-off valve; $\mathrm{R}$ - pressure regulator; SDV - safety discharge valve;

$\mathrm{M}$ - manometer; $\mathrm{T}$ - temperature recorder; $\mathrm{P}$ - pressure recorder; CT - condensate trap. 
safety systems should have actuated in sequence. Further, we will consider how it should have happened if the GDP had been in a normal operating condition.

The GDP outlet design pressure and the operating pressure of the street network was $2.0 \mathrm{kPa}$. At the pressure of $2.7 \mathrm{kPa}$ (standard settings of the equipment are provided herein), the spring safety discharge valve that is common for both lines should have actuated. In this case, gas would have been dumped into the atmosphere in the amount of up to $25 \%$ of the GDP capacity. In case of further increase in the outlet pressure to $2.9 \mathrm{kPa}$, the main line safety shut-off valve (SSV1) should have actuated, and the GDP would have automatically switched to the standby line. In case of further pressure increase, the standby line safety shut-off valve (SSV2) should have actuated at the pressure of $3.9 \mathrm{kPa}$, and gas would not have been supplied to the network anymore. The dumping of gas into the atmosphere through the safety discharge valve would have continued.

As can be seen, the situation would have ended in case of switching to the standby line, since the properly functioning pressure regulator would have started operating. But, as stated above, the GDP in question was not fully operational: by that time, it operated through the standby pressure reduction line, and both safety devices did not go off.

Speaking of the reasons behind the failure of the standby line safety shut-off valve to operate, it follows from the prosecutor's materials that its "trigger did not affect the shut-off mechanism". The authors managed to establish that the valve was just irresponsibly disabled.

According to the employees of the gas supply company, after the GDP switched to the standby line, it was often off - the safety shut-off valve actuated, and gas supply to consumers stopped. To avoid unwarranted interruptions in gas supply, the trigger of the safety shut-off valve was simply disabled physically, in the hope of sufficient safety ensured by the safety discharge valve.

\section{Issues with the safety discharge valve}

The findings of the prosecutor's investigation touch upon not the safety shut-off valve but its piping. By the end of the 1990s, hydraulic seals that acted as safety discharge valves were massively replaced by mechanical spring valves. Hydraulic seals represented simple and reliable devices ensuring protection levels 1 and 4 (25 and $100 \%$ of gas discharge, respectively). However, they required heating GDP rooms or using non-freezing liquids. Besides, the vent stack of a hydraulic seal, ensuring $100 \%$ capacity of a GDP, usually was not closed. This resulted in precipitation falling in the container, distorted physical height of device adjustment, and diluted non-freezing liquid.

However, it was $100 \%$ possibility of gas discharge that turned out to be difficult to ensure with spring devices. Technological capabilities of the industry (body casting, weight, size, etc.) significantly limited the capacity of safety discharge valves, and, according to the 2004 Polish industrial standard, devices with $100 \%$ capacity were used only in GDPs with rated consumption of up to $60.0 \mathrm{~m} 3 / \mathrm{h}$ and input pressure of more than 1.6 MPa.

In the rest of GDPs, spring safety discharge valves with the capacity of up to $25 \%$ of the rated consumption were used. Improved and safer pressure reduction lines also contributed to that. In such lines, besides the safety shut-off valve and safety discharge valve, there was also a regulator monitor. It was intended to adjust the operation of the main pressure regulator when it did not operate properly at high dynamics of input pressure. And in case of a failure of the main pressure reducer, the monitor took over its functions. Anyhow, the majority of GDPs became devoid of protection level 4 when the hydraulic seal actuated at $100 \%$ plant capacity at the pressure of $5 \mathrm{kPa}$.

It is difficult to comment on the experts' conclusion about the reduced diameter of the vent stack of the safety discharge valve. On the side of each valve, there is a hole with the internal thread for diameter corresponding to the rated capacity. The discharge pipe with such diameter is then led out above the GDP building. The height of the vent stack is calculated taking into account gas dispersion in such a way so that no explosive gas concentration formed in the lowest atmospheric layer. It is commonly at least $3.0 \mathrm{~m}$ above the ground level. It turned out impossible to establish if reduction to lesser diameter had been made. However, such reduction in diameter could not be critical to ensure gas discharge.

Data on the "siphonal", i.e. U-shaped layout of the discharge piping not protected against atmospheric precipitation, is far more important. For Polish GDPs, the piping should be L-shaped: it should be laid sideways through the wall, and then - directly upwards. We could try and explain why gas supply company employees implemented such seemingly illogical layout. No protection against moisture was commonly explained by a small diameter of the pipe. It was believed that no significant amount of precipitation could penetrate it. Nevertheless, some amount of water still penetrated the vent stack. Running down the pipe, it then entered the valve, resulting in corrosion. And in case water froze, the valve operation was completely interrupted.

Thus, the siphon could act as a condensate trap made at one's own risk, that protected the safety shutoff valve against moisture. It was assumed that when the valve actuated, water would just be driven off into the vent stack. Reduction in diameter of the discharge pipe might be caused by the use of the siphon made out of the material at hand. This is in fact a critical error made by the personnel: at a temperature below the freezing point, water in the siphon turns into an ice plug. In such case, when the safety discharge valve actuated, there was no way gas could be dumped into the atmosphere.

The situation is cleared up in the Zielona Góra city chronicle: "the first serious attack of winter" was recorded on 28.11.2010, i.e. two days before the accident. At 7 a.m., the thickness of the snow cover reached $37 \mathrm{~cm}$, and the temperature was $-11^{\circ} \mathrm{C}$. This is where the chain of reasons related to the "human factor", which led to the accident in question, ends: long-term operation of the GDP with not 
fully functioning equipment - disablement of the standby line safety shut-off valve - unlawful "siphon" layout of the discharge line - moisture entering the siphon - moisture freezing at a low temperature. The initiating factor of the accident was in fact a technical reason - a failure of the pressure regulator on the GDP standby line.

\section{Analysis of technical and administrative measures}

The prosecutor's investigation ends with determination of those guilty. For technical specialists, it is more important to determine a set of technical and administrative measures ensuring non-repetition of such accidents even at the worst combination of circumstances.

Just after the accident, operating teams of PGNiG (Polish oil and gas company) made a number of recommendations to subordinate regional offices that should have prevented such events. First, it was required to check the operation of all spring safety discharge valves. At GDPs where hydraulic seals were still installed, instrumental inspection of non-freezing liquids they were filled with was required.

Concurrently, a temporary solution was found to prevent accumulation of water in discharge lines (by means of small holes in the horizontal part of the vent stack). In case of rain, water could freely flow through such hole. It should be noted that this was in violation of the principle of preventing explosive gas concentrations from forming in the lower atmospheric layer. The hole was small, but in case of gas discharge, some gas was dumped into the atmosphere at a height of about $0.5 \mathrm{~m}$ above the ground level.

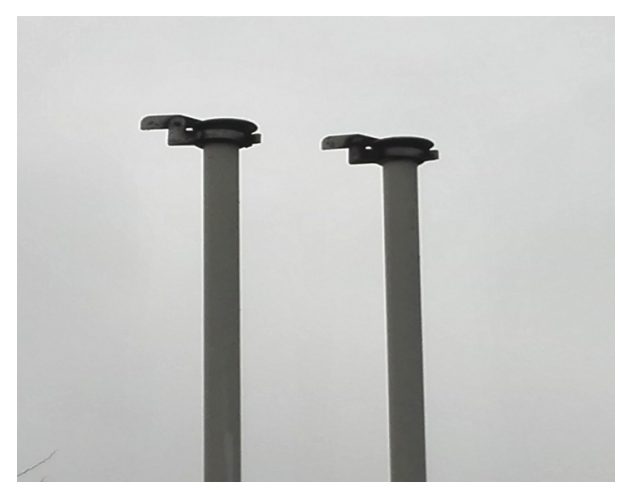

Figure 2. Standard termination of discharge and blowdown pipes at a Polish gas distribution unit plant

It was clear that it was just a half measure. That is why the next step was to structurally prevent moisture penetration in the vent stack. Hinged valves were quickly made at the end of the discharge pipe using materials at hand. The end of the pipe was often cut off at some angle. The valve opened under the pressure of the discharged gas, and after the discharge, it closed under gravity.

This should have made it possible not to make holes for water removal. However, such crude method had unavoidable consequences. Axes of the hinged joints became rusty, and sometimes they jammed, which made it difficult for the valve to open freely. And, which is worse, a blow of wind could open the valve which further remained open.

Eventually, such devices started to be manufactured in a centralized way at factories (Figure 2). The use of proper materials increased valve reliability but did not rule out the possibility of precipitation penetration in the vent stack. Dirt, feathers, leaves, moisture freezing in a hinged joint - all this can degrade pressure integrity of a closed valve.

Any Russian specialist in gas supply would wonder why their Polish colleagues did not think of a simpler and safer solution. We are referring to the bend of the vent stack termination of about $180^{\circ}$ downwards, traditionally used at our gas distribution plants (Figure 3). There are no moving parts that could "malfunction". Snow on the bend shown in the photo on the right demonstrates that there is no way for precipitation to penetrate the pipe.
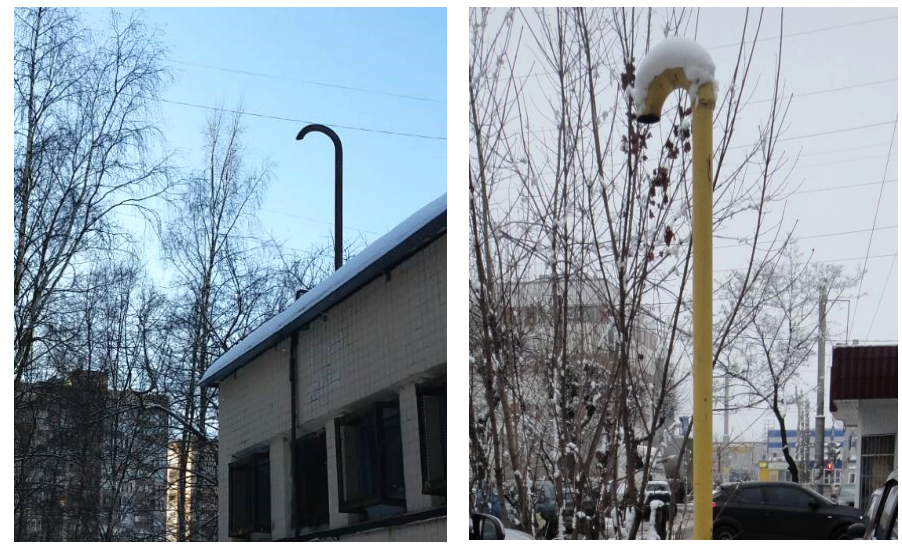

Figure 3. Standard termination of discharge pipes at Russian lowpressure GDPs.

It is not that this way was not considered by the Polish gas supply industry. But the arguments for the solution with a hinged valve look strange. As follows from the explanations by Polish colleagues, they feared that gas outflow at a great speed during discharge could move the area of explosive gas concentration downwards, i.e. to the area of the GDP devices and the working repair crew. It was even assumed that gas flow could gradually wear out the pipe bend.

We will not go into those unreasonable concerns about the pipe "wear". Instead, we will consider a simple example. For a GDP with rated consumption of $60 \mathrm{~m}^{3} / \mathrm{h}$ with the $25 \%$ capacity of the discharge line, the gas outflow rate through the vent stack with a diameter of $25-30 \mathrm{~mm}$ would be $5-8 \mathrm{~m} / \mathrm{s}$. First, the gas jet that is 1.7 times lighter than air will bend upwards instantly. Besides, together with the rate increase, the ejecting capacity of the jet also increases, and it is diffused and slowed down by air. It is virtually impossible for an explosive gas concentration to form in the lower atmospheric layer at a gas outflow height of 4.5-5 m. Otherwise, this solution could not be used in Russian gas supply.

But it is not the only question. High gas outflow during gas discharge is quite rare. In case there are standard reasons behind the safety discharge valve actuation (low dynamics of the pressure regulator, contamination of the valve seat or damage to the membrane), the valve 
operates in the pulse mode. Pressure in the discharge pipe increases gradually, the valve opens, and a small amount of gas is discharged into the atmosphere. Pressure drops drastically, the valve closes, and the pressure pulsation cycle repeats.

It shall be noted that actuation of the safety discharge valve shall be duplicated by a telemetric signal to the control unit of the gas supply company. In Western countries, in view of high dynamic characteristics of regulators, no discharge valves are usually stipulated in GDP layouts. There is only an alarm line transmitting a signal that gas pressure has increased to the set "alarm" level.

The Polish gas supply industry has developed in an inbetween way. At large plants, where gas consumption and pressure variations are smoothed out, discharge devices are not installed. At GDPs subject to drastic variations in outlet pressure, safety discharge valves with the capacity of $2 \%$ of the plant rated consumption are provided. Such device is not regarded as an element of the safety system. It just "secures" the pressure regulator if the latter does not operate properly at high dynamics of pressure variations.

\section{Origin of water in the system}

It is seemingly a rhetorical issue. The prosecutor's investigation, without any hesitation, points at the atmospheric origin of the moisture accumulated in the "unlawful" siphon of the discharge pipe. This is based on an expert opinion that, however, was accepted a priori, without any observations or proof. In other words, it was based on the following assumption: "Where else it could possibly come from?"

Nevertheless, not rejecting such possibility, the authors would like to draw the reader's attention to another source of moisture in gas supply systems, i.e. the moisture of gas itself. According to standards of any country, dry gas should be supplied to city gas distribution systems. This stereotype has led to the fact that condensate traps that had previously been installed at the lower parts of connecting gas pipes and even under each riser, became gradually disused in design practice. Their use in gas distribution pipes became limited.

In fact, we are referring to the use of preliminarily dried gas that cannot be absolutely dry in principle. Otherwise, why would gas quality standards mention the dew point temperature for moisture (Federal Agency, 2014)? The authors dedicated a whole set of researches to the issues of gas fuel quality, including its humidity (Szkarowski, $2013,2014)$. For purposes of this analysis, it is sufficient to provide an example that at the moisture content at the dew point level at the temperature of $0^{\circ} \mathrm{C}$, the absolute humidity of gas is $4.88 \mathrm{~g} / \mathrm{m}^{3}$, which corresponds to $0.61 \%$ vol. This means that a gas distribution system that supplies $1 \mathrm{mln} \mathrm{m}^{3}$ of gas, transports with it more than $6,000 \mathrm{~m} 3$ of moisture vapor.

The value of the dew point temperature in the certificates analyzed varied from +4 to $-6^{\circ} \mathrm{C}$. In reality, this figure can reach $+20^{\circ} \mathrm{C}$ in operation. This means that during wintertime, gas is inevitably saturated with moisture vapor with its subsequent condensation.

Speculations about the origin of water in the system do not change the essence of the analysis: in this case, the accident would still happen due to a combination of unfavorable technical reasons and the human factor.

\section{Conclusion}

The analysis and the need for it can be easily criticized only on the basis that the situation described is unique and simply cannot happen again. However, we would like to point out that this accident should not and even could not have happened, if not for a chain of accumulated consequences making each other stronger, similar to the domino effect. We can even mention here the butterfly effect. Of course, an unlawfully disabled valve is not the same as a light beat of butterfly's wings, but the cause and effect mechanism is unrelenting. Our task was to draw the specialists' attention to the fact that there are no such aspects in gas supply that are less or more important. Every fault or erroneous action, despite their insignificance, can lead to tragic events.

The fact that the accident considered in this paper cannot be viewed as a unique one can be proved with a fresh example. On September 14, 2018, one man died and 12 more were injured after several dozen explosions related to gas leaks in three localities near Boston, USA (Interia Fakty, 2018). 50 fire teams participated in putting out fires in Lawrence, Andover and North Andover. Hundreds of people were evacuated. According to the preliminary firemen's conclusions, the reason behind the fires was too high gas pressure. No further comments are needed. 


\section{Architecture and Engineering Volume 3 Issue 4}

\section{References}

Ansari, N., Ayatollahi, S., Riazi, M. (2017). Underground natural gas storage in a low quality gas reservoir - Produced gas quality control by rate optimization. In: Proceedings of 79th EAGE Conference and Exhibition 2017: Energy, Technology, Sustainability - Time to Open a New Chapter.

Federal Agency on Technical Regulation and Metrology (2015). GOST 5542-2014. Natural fuel gases for commercial and domestic use. Specifications. Moscow: Standartinform.

Fu, X., Li, G., Zhang, X., Qiao, Z. (2018). Failure probability estimation of the gas supply using a data-driven model in an integrated energy system. Applied Energy, 232, pp. 704-714. DOI: 10.1016/j.apenergy.2018.09.097.

Interia Fakty (2018). USA: series of gas explosions. Several dozen explosions. Available at: https://fakty.interia.pl/swiat/ news-usa-seria-wybuchow-gazu-kilkadziesiat-eksplozji,nld,2631139. (accessed on: 12.09.2018)

Kim, J.-H., Doh, D.-H., Choi, B.C. (2018). Evaluation of the ventilation safety requirements for the fuel gas supply system room of a gas-fueled vessel: Simulated leaks of methane and propane. Journal of Mechanical Science and Technology, 32 (11), pp. 5521-5532. DOI: 10.1007/s12206-018-1050-7.

Prokuratura Okrégowa (2013). The indictment against the gas distribution network master in the case of gas explosion in Zielona Góra neighbourhood. Available at: http://www.zielona-gora.po.gov.pl/index.php?id=3\&ida=9326. (accessed on: 18.10.2018)

Super Express (2010). Zielona Góra: Gas stove explosions in residential buildings. Available at: http://www.se.pl/wiadomosci/ polska/kuchenki-wybuchay-ludziom-w-domach_162460.html. (accessed on: 12.09.2018)

Szkarowski, A., Janta-Lipińska, S., Kolienko, A. (2013). Gas quality control as a tool of energy saving. Gas, Water and Sanitary Engineering, 4, pp. 146-150.

Szkarowski, A. (2014). Gas combustion. Theory, practice, ecology. (Warsaw: WNT Publishing House).

Tymchik, G.S. et al., (2018). Quality control system of well-bonded coupling fitting onto high pressure gas-main pipelines. In: Proceedings of SPIE - The International Society for Optical Engineering, 108085A. DOI: 10.1117/12.2501594.

Vasista, T.G., Alsudairi, M.A.T. (2018). Managing through computer aided quality control in oil \& natural gas industry project sites. Journal of Advanced Research in Dynamical and Control Systems, 10 (4), pp. 896-905.

Wang, Y., Yang, S. (2018). Proposal and thermodynamic analysis of a combined open-cycle absorption heat pump and thermal desalination system driven by high-humidity exhaust gas. Desalination, 448, pp. 93-102. DOI: 10.1016/j.desal.2018.10.003. 